\title{
Research on Multi-objective Dynamic Task Scheduling of Cross-aisles Multi-layer Shuttle Truck Storage System Based on Elite Strategy
}

\author{
Ran ZHANG ${ }^{1}$, Zhuan WANG ${ }^{1}$, , Yu-xin $A N^{1}$ \\ ${ }^{1}$ School of mechanical engineering, University of Science and Technology Beijing, China.
}

\begin{abstract}
In order to improve the operating efficiency of the cross-aisles shuttle truck system and the responsiveness to the disturbance of the return task, this paper first proposes a dynamic task scheduling process for the shuttle truck, using an event trigger mechanism. Each time a warehouse return task is generated, the corresponding scheduling optimization algorithm is triggered according to the current task combination mode to re-optimize the task sequence. Secondly, a task sequencing model is established under three combined task modes: full outbound / full return tasks, task mode with in-transit return tasks under the compound operation mode, task mode without in-transit return tasks under the compound operation mode. A fast non-dominated sorting genetic algorithm scheduling algorithm with elite strategy for compound operation mode is proposed. Finally, MATLAB was used to compile the simulation experiment system. The simulation analysis results show that the method can effectively improve the system's operating efficiency, which verifies the feasibility and effectiveness of the scheduling method.
\end{abstract}

\section{Introduction}

The multi-layer shuttle truck system can greatly improve the efficiency of entering and leaving the warehouse. The traditional multi-layer shuttle truck system has only one shuttle truck for each aisle in the same floor. The crosslayer shuttle truck system studied in this paper shares a shuttle truck in the same floor's multiple aisles. The shuttle truck can change the aisles according to the task to complete the operation tasks. In the study of multi-layer shuttle truck systems, scholars analyze the system's picking task process and the impact of the number of shuttle trucks on the system operating efficiency, solve the configuration optimization model [1-3]. Quantitative simulation experiments were performed on the structure of the shuttle truck using the dynamic programming and other algorithms to optimize the path of the model scheduling [4-5]. Khojasteh Ghamari and others used genetic algorithm, particle swarm algorithm, simulated annealing algorithm, etc. to optimize the storage location allocation and picking path [6-10]. Ran Wenxue merged the taboo search algorithm and pareto ideas, and matched tasks whose inbound and outbound of the warehouse are relatively closer to obtain an effective scheduling optimization scheme [11].

This paper builds a dynamic scheduling model of the cross-aisles shuttle truck storage system, and considers the return tasks of the warehouse. The paper sets three scheduling modes according to the different task combinations, and chooses different optimization goals and solving methods. During the operation of the system, whenever a new return task is added to the order pool, a

\footnotetext{
a Corresponding author: wangzhuan@ustb.edu.cn
}

scheduling mode corresponding to the task type of the current order pool is triggered, and the task sequence of the shuttle truck is adjusted in time. Finally, an example is used to verify the feasibility of this method.

\section{Dynamic task scheduling process of cross-aisles shuttle truck}

\subsection{Dynamic task scheduling problem of cross- aisles shuttle truck}

The cross-aisles shuttle truck storage system consists of a shuttle truck, a hoist, a transfer truck, a shelving system, a conveying system, and a picking station, as shown in Figure 1.

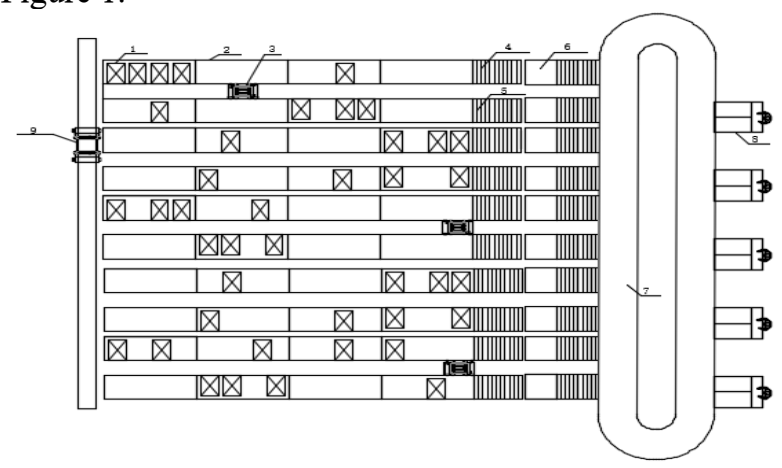

1.Cargo unit 2. Shelf 3. Shuttle truck 4. Storage buffer zone

5. Storage buffer zone 6. Hoist 7. Conveyor line

8. Sorting station 9 . Reloading truck

Figure 1. The cross-aisles shuttle truck storage system. 
In the cross-aisles shuttle truck storage system, the order in which the shuttle truck executes tasks determines the performance indicators. When the inventory amount of a certain outbound task is greater than the outbound amount, a return task will be generated after the outbound task is completed, which will affect the task queue. Therefore, this paper considers the impact of the return tasks. During the operation of the cross-aisles shuttle truck storage system, whenever a new return task is added to the order pool, the task sequence of the shuttle truck needs to be adjusted in time. The task queue in the current order pool is re-optimized and sorted to form a new task sequencing scheme, thereby reducing the shuttle truck's running time and waiting time for return tasks, and improving the efficiency of goods outbound and inbound.

During the operation of system, when the scheduling mechanism needs to be triggered to optimize the task sequence, three different task combination modes will appear in the order pool. For different modes, different optimization objectives and solving methods are used. The three task combination modes are as follows:

(1) Full outbound / full return tasks mode: The shuttle truck order pool is all outbound tasks or return tasks. At this time, the shuttle truck adopts the single operation mode.

(2) Task mode without in-transit return tasks under the compound operation mode: There are both outbound tasks and return tasks in the order pool of the shuttle truck, but there are no in-transit return tasks. That is, in the current situation, for the goods that have been out of the warehouse, the tasks that need to be returned to the warehouse because the amount of outbound is lower than inventory have all reached the task pool and are waiting for the return operation.

(3) Task mode with in-transit return tasks under the compound operation mode: As the task progresses, the return task is generated, and the order pool of the shuttle truck is changed. There are outbound tasks and return tasks, and there are also in-transit return tasks.

Based on this, the dynamic scheduling model of the cross-aisles shuttle truck storage system will set three corresponding scheduling optimization methods and make the following reasonable assumptions:

(1) For outbound tasks with inventory quantity higher than outbound quantity, a return task will be generated after the outbound sorting is completed.

(2) The number of buffers is two, and a maximum of two bins can be stored, which can be empty.

(3) At most one bin is stored in each cargo storage space, which can be empty.

(4) Does not consider the impact of system equipment failure on the model.

\subsection{Dynamic task scheduling process of cross- aisles shuttle truck}

The dynamic task scheduling process of the shuttle truck is shown in Figure 2. After the batch tasks are issued, the corresponding scheduling optimization algorithm needs to be selected according to the task combination type in the order pool, the shuttle truck task order is optimized, and the task execution sequence is obtained. The shuttle truck completes the loading task in sequence according to the task execution sequence. When a new return task is generated, the corresponding optimization scheduling method is triggered according to the current task combination type, and the remaining tasks are optimized and sorted again, and the cycle is completed until all tasks are completed.

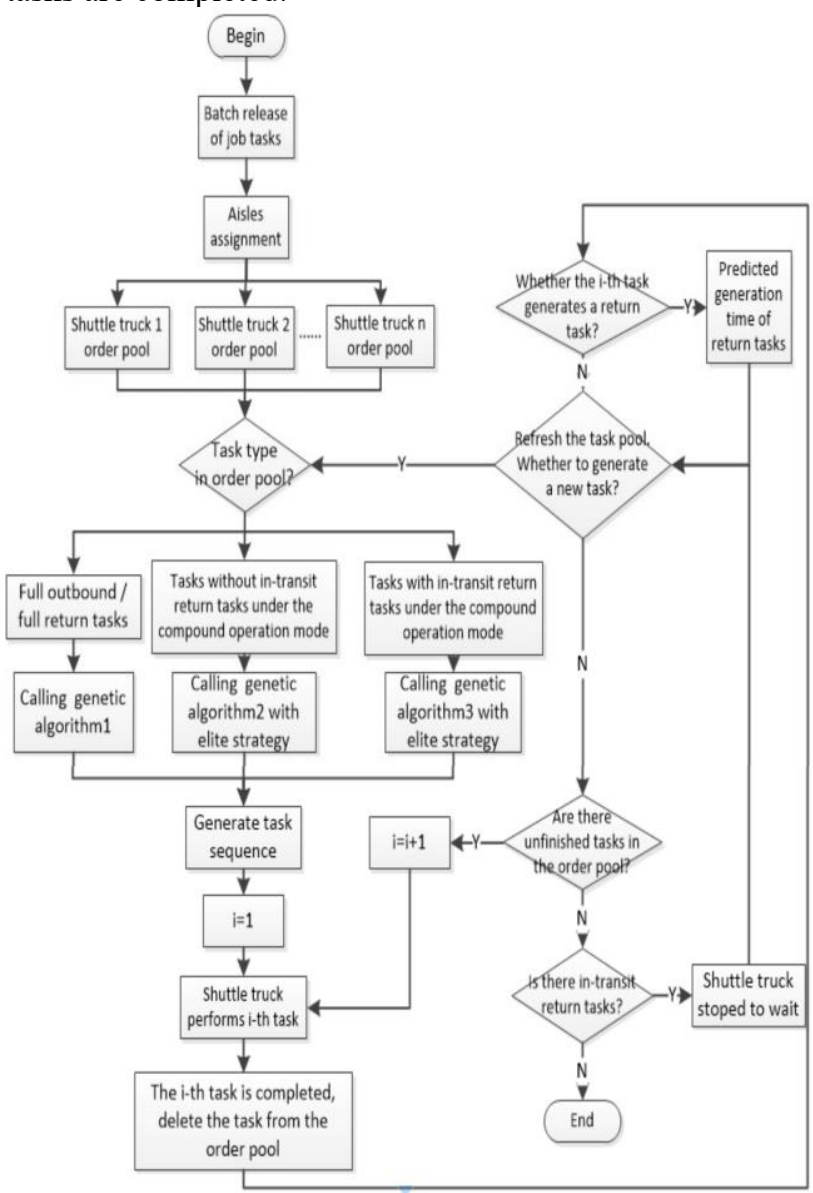

Figure 2. Dynamic task scheduling process of cross-aisles shuttle truck.

In the dynamic scheduling of the shuttle truck tasks, an event trigger mechanism is used. Each time a task is returned to the warehouse, the corresponding scheduling optimization algorithm is triggered according to the current task combination mode to re-optimize the task sequence. It includes three scheduling optimization methods. When tasks in the order pool are all outbound or all return to the warehouse, the genetic algorithm is used to optimize the task sequence with the goal of the shortest total task completion time. When there are both outbound and return tasks in the order pool, determine whether there are return tasks in-transit. If there are, optimize the task sequence with the goals of the shortest waiting time for return tasks and the maximum number of tasks completed in the forecast period; Otherwise, optimize the task sequence with the goals of the shortest total task completion time and waiting time for return tasks.

\section{Dynamic task scheduling model of cross-aisles shuttle truck}




\subsection{Model parameter settings}

In the cross-aisles shuttle truck system, a row of shelves is provided with $d$ cargo storage spaces, and each cargo storage space has a length $q$, a width $p$, and an aisle width $o$. There are $m$ aisles on a certain floor, the aisle number is $R i(i=1,2,3, \ldots, m)$; there are $n$ shuttle trucks, the shuttle truck number is $C j(j=1,2,3, \ldots, n)$. The position coordinate of the $\mathrm{j}$-th shuttle truck is $\left(X_{\mathrm{C}_{\mathrm{j}}}, Y_{\mathrm{C}_{\mathrm{j}}}\right), X_{\mathrm{C}_{\mathrm{j}}}$ is the aisle number where the shuttle truck is located, and $Y_{\mathrm{C}_{\mathrm{j}}}$ is the corresponding cargo storage space number of the shuttle truck. The number of shuttle truck's j-th task is $N_{\mathrm{C}_{\mathrm{j}}}$, where the number of outbound tasks is $A_{\mathrm{C}_{\mathrm{j}}}$, the number of return tasks is $B_{C_{j}}$, and the corresponding cargo storage space coordinates to the $\mathrm{s}$-th task is $\left(X_{s}^{0}, Y_{s}^{0}\right)\left(\mathrm{s} \in N_{\mathrm{C}_{\mathrm{j}}}\right)$. The coordinates of the transfer vehicle is $\left(X_{\mathrm{C}}, Y_{\mathrm{C}}\right), X_{\mathrm{C}}$ is the number of the aisle where the transfer vehicle is located, and $Y_{\mathrm{C}}$ is the virtual cargo storage space number corresponding to the transfer vehicle $Y_{\mathrm{C}}=\mathrm{d}+1$.

Taking the $\mathrm{j}$-th shuttle truck as an example, the parameters of the cross-aisles shuttle truck system are shown in Table 1.

Table 1. Model variables for task scheduling of cross-aisles shuttle truck system.

\begin{tabular}{|c|c|c|}
\hline Type & Symbol & Variable Description \\
\hline $\begin{array}{l}\text { Decision } \\
\text { variables }\end{array}$ & $X_{a b}$ & $X a b=\left\{\begin{array}{l}0, \text { The next task of task a isn't task } b \\
1, \text { The next task of task a is task } b\end{array}\right.$ \\
\hline \multirow{10}{*}{$\begin{array}{l}\text { Intermediate } \\
\text { variable }\end{array}$} & $t_{a b}$ & $\begin{array}{l}\text { The shuttle truck starts from the end of } \\
\text { task } a \text {, and the time to complete task } b \text { is } \\
\qquad t_{\mathrm{ab}}\end{array}$ \\
\hline & $t_{c j}^{k}$ & $\begin{array}{l}\text { Total free time of the } \mathrm{j} \text {-th shuttle truck is } \\
\qquad t_{c_{j}}^{k}=\sum_{\mathrm{a}=0}^{N C_{j}}\left(\begin{array}{c}t_{\text {start }} \\
t_{\text {end }}\end{array}\right) .\end{array}$ \\
\hline & $t_{c j}$ & $\begin{array}{l}\text { The total time for the } \mathrm{j} \text {-th shuttle truck to } \\
\text { complete all tasks is } t_{c_{j}}=\sum_{\substack{a=0 \\
N c_{j}}}^{\sum_{b=1}^{N c_{j}}} X_{a b}{ }^{*} t_{a b}+t_{c j}^{k}\end{array}$ \\
\hline & $t_{\text {start }}^{s}$ & Start time of the s-th task. \\
\hline & $t_{\text {end }}^{s}$ & End time of the s-th task. \\
\hline & $t_{\text {calc }}^{s}$ & $\begin{array}{c}\text { Generation time of the return prediction } \\
\text { of the s-th task. }\end{array}$ \\
\hline & $t_{\text {true }}^{s}$ & Actual return time of the s-th task. \\
\hline & $t_{\text {load }}^{s}$ & $\begin{array}{l}\text { The time when the s-th return task enters } \\
\text { the task queue. }\end{array}$ \\
\hline & $t_{s}^{w}$ & $\begin{array}{l}\text { The waiting time for the s-th return task } \\
\text { is } t_{s}^{w}=t_{\text {start }}^{s}-t_{\text {load }}^{s}\end{array}$ \\
\hline & $t_{\text {calc }}$ & $\begin{array}{l}\text { The set of prediction times for all return } \\
\text { tasks. }\end{array}$ \\
\hline
\end{tabular}

\begin{tabular}{|c|c|c|}
\hline \multirow{4}{*}{ Fixed value } & $T_{c}$ & $\begin{array}{c}\text { Time for the shuttle truck to get on and } \\
\text { get off the transfer vehicle. }\end{array}$ \\
\cline { 2 - 3 } & $T_{h}$ & $\begin{array}{c}\text { Picking and unloading time of the } \\
\text { shuttle truck. }\end{array}$ \\
\cline { 2 - 3 } & $V_{1}$ & Speed of the shuttle truck \\
\cline { 2 - 3 } & $V_{2}$ & Speed of the transfer vehicle \\
\hline
\end{tabular}

(1) Running time of cross-aisles shuttle truck system

The running time of the entire cross-aisles shuttle truck system is $\mathrm{T}=\max t_{C_{j}}=\max \left\{t_{C_{1}}, t_{C_{2}}, \ldots, t_{C_{n}}\right\}$. The longest time of the shuttle truck to complete the task is taken as the running time of the entire system.

(2) Prediction time of return tasks $t_{\text {calc }}^{s}$

Generation time of the return prediction of the s-th task: The time when the return task is generated $t_{\text {end }}^{s^{\prime}}+$ hoisting time $t_{l}+$ sorting time + conveyor line transportation time $t_{r}$.

$t_{\text {calc }}^{s}=t_{\text {end }}^{s^{\prime}}+$ outbound quantity * picking time of single item $+t_{l}+t_{r}$

(3) Execution time of each task $t_{\mathrm{ab}}$

When task $b$ is executed, the initial position of the shuttle truck is the end position of the previous task $a$, and the formula for the execution time $t_{\mathrm{ab}}$ of task $b$ is as follows:

(1) If task $b$ is the outbound task, and task $b$ is in the same aisle with the shuttle truck, that is, task $a$ and task $b$ are in the same aisle.

$$
\begin{aligned}
& t_{a b}=t_{s}^{1}+t_{s}^{2}+2 T_{h} \\
& t_{s}^{1}=\frac{\left|Y_{C_{j}}-Y_{s}^{0}\right| * q}{V_{1}} \\
& t_{s}^{2}=\frac{Y_{s}^{0} * q}{V_{1}}
\end{aligned}
$$

(1) indicates that the execution time $t_{\mathrm{ab}}$ of the outbound task that does not need to cross the aisles consists of three time periods, the first period is represented by (2), $t_{s}^{1}$ is the time for the shuttle truck to travel from the current position to the outbound cargo storage position; and the second period is (3), $t_{s}^{2}$ the time for the shuttle truck to pick up the goods from the outbound cargo storage position and go to the outbound buffer; and the third time period $T_{h}$ is the fixed picking and unloading time of the shuttle truck.

(2) If task $b$ is the outbound task, and task $b$ is not in the same aisle as the shuttle truck, that is, task $a$ and task $b$ are not in the same aisle.

$$
\begin{aligned}
t_{a b} & =t_{s}^{1}+t_{s}^{2}+2 T_{h} \\
t_{s}^{1} & =t_{s}^{11}+t_{s}^{12}+t_{s}^{13}+2 T_{c} \\
t_{s}^{11} & =\frac{\left|Y_{c}-Y_{C_{j}}\right| * q}{V_{1}}
\end{aligned}
$$




$$
\begin{gathered}
t_{s}^{12}=\frac{\left|X_{c}-X_{C_{j}}\right| *(2 * p+0)+\left|X_{s}^{0} / 2-X_{C_{j}}\right| *(2 * p+0)}{V_{2}} \\
t_{s}^{13}=\frac{\left|Y_{c}-Y_{s}^{0}\right| * q}{V_{1}} \\
t_{s}^{2}=\frac{Y_{s}^{0} * q}{V_{1}}
\end{gathered}
$$

(4) indicates that the execution time of the outbound task that needs to cross the aisles consists of three time periods, the first period is indicated by (5), the second period is indicated by (9), and the third period is $T_{h}$. Among them, $t_{s}^{1}, t_{s}^{2}$, and $T_{h}$ have the same meanings as in (1).

$t_{s}^{1}$ is composed of four parts. (6) represents the time of the shuttle truck from the current position to the transfer port of the current aisle, and (7) represents the time that the transfer vehicle from the current location to the aisle of the shuttle truck and the shuttle truck is brought to the transfer port of the aisle where the outbound task is located. (8) is the time for the shuttle truck to move from the transfer port to the outbound cargo storage position, and $T_{c}$ is the fixed time for the shuttle truck to get on and get off the transfer vehicle.

(3) If task $b$ is a return task, and task $b$ is in the same aisle as the shuttle truck, that is, task $a$ and task $b$ are in the same aisle.

$$
\begin{aligned}
& t_{a b}=t_{s}^{1}+t_{s}^{2}+2 T_{h} \\
& t_{s}^{1}=\frac{Y_{c_{j}} * q}{V_{1}} \\
& t_{s}^{2}=\frac{Y_{s}^{0} * q}{V_{1}}
\end{aligned}
$$

(10) represents the execution time of the warehouse return task that does not need to cross the aisles, it consists of three time periods. The first period is indicated by (11), $t_{s}^{1}$ is the time when the shuttle truck travels from the current position to the return buffer zone; the second period is indicated by (12), $t_{s}^{2}$ is the time for the shuttle truck from the return buffer zone to the cargo storage position; the third period $T_{h}$ is the fixed picking and unloading time of the shuttle truck.

(4) If task $b$ is a return task, and task $b$ is not in the same aisle as the shuttle truck, that is, task a and task $b$ are not in the same aisle.

$$
\begin{aligned}
& t_{a b}=t_{s}^{1}+t_{s}^{2}+2 T_{h} \\
& t_{s}^{1}=t_{s}^{11}+t_{s}^{12}+t_{s}^{13}+2 T_{c} \\
& t_{s}^{11}=\frac{\left|Y_{c}-Y_{C_{j}}\right| * q}{V_{1}} \\
& t_{s}^{12}=\frac{\left|X_{c}-X_{C_{j}}\right| *(2 * p+0)+\left|X_{s}^{0} / 2-X_{C_{j}}\right| *(2 * p+0)}{V_{2}} \\
& t_{s}^{13}=\frac{Y_{c} * q}{V_{1}}
\end{aligned}
$$

$$
t_{s}^{2}=\frac{Y_{s}^{0} * q}{V_{1}}
$$

(13) represents the execution time of the warehouse return task that needs to cross the aisles, which consists of three time periods. The first period is represented by (14), the second period is represented by (18), and the third period is $T_{h}$. Among them, $t_{s}^{1}, t_{s}^{2}$, and $T_{h}$ have the same meanings as in (3).

$t_{s}^{1}$ consists of four parts. (15) indicates the time of the shuttle truck from the current position to the transfer port of the current aisle, (16) indicates the time that the transfer vehicle from the current location to the aisle of the shuttle truck and the shuttle truck is brought to the transfer port of the aisle where the return task is located. (17) is the time for the shuttle truck to move from the transfer port to the return buffer zone, and $T_{\mathrm{c}}$ is the fixed time for the shuttle truck to get on and get off the transfer vehicle.

\subsection{Sequencing model of full outbound / full return tasks}

For a certain shuttle truck, when the order pool has a single task status, that is, when tasks are all outbound or return tasks, the number of tasks is $N_{\mathrm{C}_{\mathrm{j}}}$, and the different task queues will cause different distances and running times of the shuttle truck. Change the task execution order of the shuttle truck to reduce the total task completion time.

Objective function:

$$
\operatorname{minf}=\min t_{c_{j}}=\min \left(\sum_{a=0}^{N c_{j}} \sum_{b=1, b \neq a}^{N c_{j}} X_{a b} * t_{a b}+t_{c_{j}}^{k}\right)
$$

Restrictions:

$$
\begin{gathered}
\sum_{a=0, a \neq b}^{N c_{j}} X_{a b}=1, \quad \mathrm{~b}=1,2,3, \ldots, \mathrm{Nc}_{\mathbf{j}} ; \\
\sum_{a=0}^{N c_{j}} \sum_{b=1, b \neq a}^{N c_{j}} X_{a b}=N c_{j}, \mathrm{a}=0,1,2,3, \ldots, \mathrm{Nc}_{\mathrm{j}} ; \quad \mathrm{b}=1,2,3, \ldots, \mathrm{Nc}_{\mathrm{j}} ; \\
X_{a b} \in\{0,1\}
\end{gathered}
$$

\subsection{Task sequencing model in compound operation mode without in-transit return tasks}

In the cross-aisles shuttle truck system, the $\mathrm{j}$-th shuttle truck has $A c_{j}$ outbound tasks and $B c_{j}$ return tasks, and there are no in-transit return tasks. The total number of tasks is $N c_{j}$. The task sequence is different, the total running time of the shuttle truck and the waiting time for the return task will be also different. By change the task execution order of the shuttle truck, the total completion time of the tasks and the waiting time for return tasks are reduced. The model is a two-objective optimization model.

Objective function: 


$$
\begin{gathered}
\operatorname{minf} 1_{1}=\min t_{c_{j}}=\min \left(\sum_{a=0}^{N c_{j}} \sum_{b=1, b \neq a}^{N c_{j}} X_{a b} *_{a b}+t_{c_{j}}^{k}\right) \\
\operatorname{minf}_{2}=\min _{h=1}^{B c_{j}} t_{h}^{w}=\sum_{h=1}^{B c_{j}}\left(t_{\text {start }}^{h}-t_{\text {load }}^{h}\right)
\end{gathered}
$$

(23) indicates that the task sequence optimization is performed with the goal of the shortest total completion time for all tasks; (24) indicates that the task sequence optimization is performed with the goal of the shortest total waiting time of all return tasks.

Restrictions:

$$
\begin{gathered}
\sum_{\substack{a=0, a \neq b \\
N c_{j}}} X_{a b}=1, \quad \mathrm{~b}=1,2,3, \ldots, \mathrm{Nc}_{\mathrm{j}} ; \\
\sum_{a=0 b=1, b \neq a}^{N c_{j}} \sum_{\substack{N c_{j} \\
C_{a b}}} X_{a b}=N c_{j}, \quad \mathrm{a}=0,1,2,3, \ldots, \mathrm{Nc}_{\mathrm{j}} ; \mathrm{b}=1,2,3, \ldots, \mathrm{Nc}_{\mathrm{j}} \\
X_{a b} \in\{0,1\}
\end{gathered}
$$

\subsection{Task sequencing model in compound operation mode with in-transit return tasks}

In the cross-aisles shuttle truck system, the $\mathrm{j}$-th shuttle truck has $A_{C j}$ outbound tasks and $B_{C j}$ return tasks at time $T a$, and includes in-transit return tasks. It is predicted that in the Td-th second, there will generate a return task and enter the order pool. The different task queues will cause the distance, running time and waiting time of the shuttle truck to be different. Adjust the order of the task queues so that complete tasks as many as possible before the predicted return task is generated, and shorten the waiting time of return tasks. The model is a two-objective optimization model.

Process parameter:

$$
\begin{aligned}
\mathrm{t}_{\text {calc }} & =\operatorname{renew}\left\{\mathrm{t}_{\text {calc }}\right\} \\
\mathrm{T}_{\mathrm{d}} & =\min \left\{\mathrm{t}_{\text {calc }}\right\}
\end{aligned}
$$

Objective function:

$$
\begin{gathered}
\underset{\max f_{1}}{ }=\operatorname{maxnum}\left[T_{a}, T_{d}\right]=\operatorname{maxnum}\left[T_{a}<t_{C_{j}}<T_{d}\right] \\
=\operatorname{maxnum}\left[T_{a}<\sum_{a=0}^{N c_{j}} \sum_{b=1, b \neq a}^{N c_{j}} X_{a b} *_{a b}+t_{c_{j}}^{k}<T_{d}\right] \\
\min f_{2}=\min \sum_{h=1}^{N} t_{h}^{w}=\sum_{h=1}^{N}\left(t_{\text {start }}^{h}-t_{\text {load }}^{h}\right)
\end{gathered}
$$

(28) means updating the predicted set. Delete the task rows in the set that are smaller than the current moment and the predicted task rows that have actually returned to the warehouse. These task rows have expired and have no predictive value.

(29) represents selecting the minimum value $T_{d}$ in the set of return prediction times. $T_{d}$ represents the predictable earliest generation time of return task.

(30) is the objective function 1, which indicates that the scheduling order is optimized with the goal of the maximum number of tasks completed in the time period $\left[T_{a}, T_{d}\right] . T_{a}$ represents the current moment; $T_{d}$ represents the minimum value of the prediction time for return task, that is, the predicted time when the earliest return task is generated.
(31) is the objective function 2, which means to optimize the scheduling order of tasks with the goal of the shortest waiting time of all return tasks in the order pool.

Restrictions:

$$
\begin{gathered}
\sum_{a=0, a \neq b}^{N c_{j}} X_{a b}=1, \quad \mathrm{~b}=1,2,3, \ldots, \mathrm{Nc}_{\mathrm{j}} ; \\
\sum_{a=0}^{N c_{,}, N c_{j}, b \neq a} \sum_{a b}=N c_{j}, \mathrm{a}=0,1,2,3, \ldots, \mathrm{Nc}_{\mathrm{j}} ; \mathrm{b}=1,2,3, \ldots, \mathrm{Nc}_{\mathrm{j}} ; \\
X_{a b} \in\{0,1\}
\end{gathered}
$$

\section{Solving method for dynamic task scheduling of cross-aisles shuttle truck based on elite strategy}

In the full outbound / full return task operation mode, with the goal of the minimum total task completion time, the basic genetic algorithm is used to solve the problem. Natural coding is used to randomly generate the initial population and the roulette mechanism is selected. The fitness function is determined by the total time of task. When the maximum number of iterations is reached, the chromosome with the highest fitness is selected as the optimal solution of the algorithm, that is, the optimal task queue order. The crossover and mutation strategy of the basic genetic algorithm is consistent with the genetic algorithm with the elite strategy below.

This paper focuses on the two scheduling modes under the compound operation mode, and uses the fast non-dominated sorting genetic algorithm with elite strategy to solve. The fast non-dominated sorting method can reduce the complexity of the algorithm, the congestion degree and comparison operator can maintain the diversity of the population, and the introduction of the elite strategy can expand the sample space, quickly improve the population level, effectively shorten the solution time, and make the solution result as optimal as possible.

(1) Encoding

For the task scheduling model of the cross-aisles shuttle truck system, the chromosome code is the natural code, the chromosome is the task sequence, and the individual is $\left(r_{1}, r_{2}, r_{3}, \ldots, r_{n}\right)$, where $r_{i}(1 \leq i \leq n)$ represents a certain gene in the chromosome, which is the execution order of the i-th task.

(2) Fitness function

$\operatorname{minf}_{1}=\operatorname{mint}_{C_{j}}, t_{c_{j}}$ is the total completion time of the $\mathrm{j}$-th shuttle truck. (Suitable for model 2).

$$
\operatorname{minf}_{2}=\min \sum_{j=1}^{n} \sum_{\gamma_{2}=1}^{B c_{j}} t_{h}^{w} \text {, which is the sum of the }
$$

waiting time of all the return tasks. (For models 2, 3)

$\operatorname{maxf}_{2}=\operatorname{maxnum}\left[T_{a}, T_{d}\right]$, is the number of tasks completed in the time period $\left[T_{a}, T_{d}\right]$. (For model 3)

(3) Stratification 
Find the Pareto optimal solution set of the objective function in the population. First, based on the value of the multi-objective function, the first layer Pareto optimal solution $B_{1}$ is found. After removing $B_{1}$, the second layer optimal solution set $B_{2}$ is determined among the remaining individuals in the population. Cycle until all individuals in the population are stratified. The virtual fitness values of individuals in the same layer are equal. The smaller the layer number is, the larger the virtual fitness value is.

(4) Congestion operator

When the layer numbers of chromosomes are consistent, the crowded distance of chromosomes should be compared to determine which chromosome is more suitable.

k.distance $=(\mathrm{f}(\mathrm{k}+1) \cdot \mathrm{m}-\mathrm{f}(\mathrm{k}-1) \cdot \mathrm{m}) /(\max \cdot \mathrm{m}-\operatorname{minf} \cdot \mathrm{m})$

\section{(5) Elite strategy}

Parents and offspring are determined by layering and crowding distance. For individuals in the same population, according to the requirements of the number of layers from small to large and crowded distance from large to small, the individuals are placed in the set in order until the elite retention policy is met.

(6) Cross strategy

Adopt PMX-like cross method. Select two chromosomes $A$ and $B$, and randomly select two points in $A$ and $B$. The middle part is the cross section of the chromosome. The $A$ and $B$ 's cross sections are placed at the front of each other's chromosomes to form chromosomes $A_{1}$ and $B_{l}$. Remove the duplicated gene items in each chromosome, and get the crossed individuals $A_{2}$ and $B_{2}$.

(7) Variation

An inversion mutation operator is used. Select two random points of chromosome $A$, the middle is the variation interval, and rewrite the mutation interval genes in reverse order into the mutation region, effectively improving the global optimization of the genetic algorithm.

(8) Parameter settings and termination conditions

This paper takes $P c=0.8$; mutation probability $P m=$ 0.2 . Iteration number termination method is used. When the maximum number of iterations is reached, the chromosome with the highest fitness is selected as the optimal solution of the algorithm, that is, the optimal task queue order. For the pareto solution set, in model 2, the solution with the shortest total task completion time is preferred; in model 3, the solution with the most number of tasks completed in the $\left[T_{a}, T_{d}\right]$ time period is preferred; if there are multiple solutions that are the same, choose the individual with the least return tasks waiting time.

\section{Instance verification}

\subsection{Experimental parameters and schemes}

This article takes an e-commerce logistics center as an example. The automated stereoscopic warehouse is set up with 10 floors, 12 rows, and 80 columns. In this paper, the fifth layer is selected as the simulation module, and the number of the shuttle truck is set to 3 . The main parameter settings are shown in Table 2.

Table 2. Parameter settings table.

\begin{tabular}{|c|c|c|c|}
\hline Parameter & Value & Parameter & Value \\
\hline $\begin{array}{c}\text { Cargo storage } \\
\text { space length }\end{array}$ & $0.4 \mathrm{~m}$ & $\begin{array}{c}\text { Running speed of } \\
\text { shuttle truck }\end{array}$ & $2 \mathrm{~m} / \mathrm{s}$ \\
\hline $\begin{array}{c}\text { Cargo storage } \\
\text { space width }\end{array}$ & $0.6 \mathrm{~m}$ & $\begin{array}{c}\text { Running speed of } \\
\text { transfer vehicle }\end{array}$ & $2 \mathrm{~m} / \mathrm{s}$ \\
\hline Aisle width & $0.8 \mathrm{~m}$ & $\begin{array}{c}\text { Picking and unloading } \\
\text { time of shuttle truck }\end{array}$ & $5 \mathrm{~s}$ \\
\hline $\begin{array}{c}\text { Time for shuttle } \\
\text { truck to get on } \\
\text { and get off the } \\
\text { transfer vehicle. }\end{array}$ & $2 \mathrm{~s}$ & $\begin{array}{c}\text { Time of sorting single } \\
\text { goods }\end{array}$ & $2 \mathrm{~s}$ \\
\hline
\end{tabular}

In order to verify the effectiveness of the optimization algorithm, this paper compares the optimization results with the results of traditional scheduling methods. Select the order data of 70 orders, 90 orders, 120 orders, and 150 orders in the e-commerce logistics center, and under different orders operation, count the two scheduling methods' total operation time of the shuttle truck and the average waiting time of the return tasks.

\subsection{Experimental results and conclusions}

For the order data of 70 orders, 90 orders, 120 orders, and 150 orders in the e-commerce logistics center, MATLAB simulation verification was performed by using the optimized scheduling algorithm and the traditional scheduling algorithm. The total running time and return of the shuttle truck under the two methods. The average waiting time of return tasks is shown in Table 3, and the optimization results are shown in Figures 3 and 4.

Table 3. Comparison of operation time under two methods.

\begin{tabular}{|c|c|c|c|}
\hline \multirow{2}{*}{$\begin{array}{c}\text { Number } \\
\text { of orders }\end{array}$} & $\begin{array}{c}\text { Statistical } \\
\text { parameters }\end{array}$ & $\begin{array}{c}\text { Optimized } \\
\text { scheduling } \\
\text { method }\end{array}$ & $\begin{array}{c}\text { Traditional } \\
\text { scheduling } \\
\text { method }\end{array}$ \\
\hline \multirow{2}{*}{ 70orders } & $t_{c_{j}}$ & 674.40 & 804.40 \\
\cline { 2 - 4 } & $t^{w}$ & 112.20 & 166.51 \\
\hline \multirow{2}{*}{ 90orders } & $t_{c_{j}}$ & 884.40 & 1135.00 \\
\cline { 2 - 4 } & $t^{w}$ & 117.00 & 179.99 \\
\hline \multirow{2}{*}{ 120orders } & $t_{c_{j}}$ & 1094.00 & 1521.00 \\
\cline { 2 - 4 } & $t^{w}$ & 153.85 & 215.03 \\
\hline \multirow{2}{*}{ 150orders } & $t_{c_{j}}$ & 1451.40 & 2044.20 \\
\cline { 2 - 4 } & $t^{w}$ & 160.11 & 226.68 \\
\hline
\end{tabular}




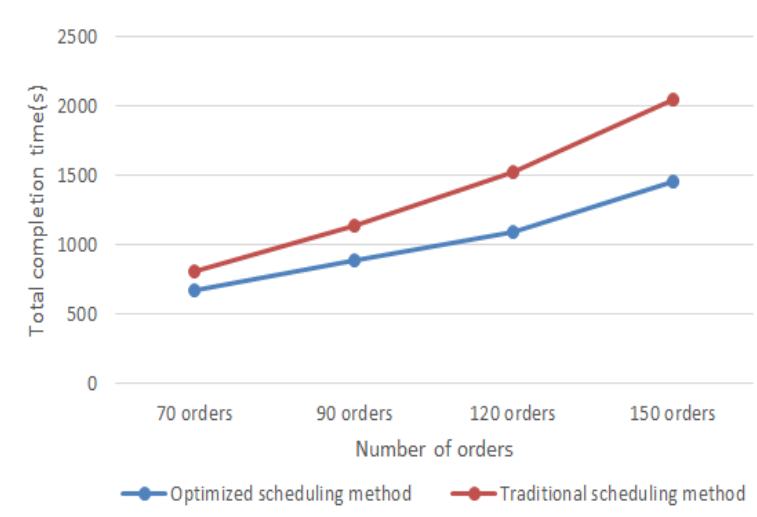

Figure 3. Comparison of total operation time under two methods.

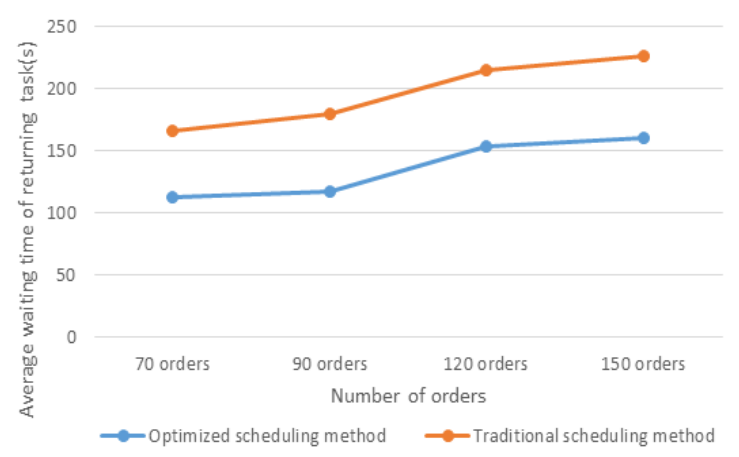

Figure 4. Comparison of average waiting time of return task under two methods.

The dynamic task scheduling model of the cross-aisle shuttle truck is simulated with multiple groups of experimental schemes, and the multi-objective solution method based on the elite strategy is used to optimize the scheduling. Compared with the traditional scheduling method, the optimization effect is obvious.

(1) The total completion time of the shuttle truck operation is effectively reduced, and the optimization rate is between $16 \%-25 \%$.

(2) The average waiting time of the return tasks is significantly reduced, and the optimization rate is between $28 \%$ and $35 \%$.

(3) With the increase of the number of tasks, the optimization rate of the total completed operation time of the shuttle truck is greater and the optimization effect is also more obvious.

(4) With the increase of the number of tasks, the average waiting time optimization rate of the return tasks fluctuated little and remained basically at $30 \%$.

\section{References}

1. Jiao Yu. Research on Multi-dimensional Intelligent Dynamic Scheduling Model of Multilayer Shuttle Truck in Automated Stereo Warehouse. Yunnan University of Finance and Economics (2016).

2. Guozhen Chen. Optimizing the Storage System Configuration of Multilayer Shuttle Truck. Shandong University (2016).
3. Wenkai Ma, Yaohua Wu, Yingying Wu. Research on Multi-layer Shuttle Truck Storage System Based on Evolutionary Algorithms. Journal of Mechanical Engineering, 55 (8): 216-224 (2019).

4. Kangkang Le. Study on the Optimal Dispatching Control of Mother-son Shuttle Truck in Threedimensional Storage System. Nanjing Forestry University (2016).

5. Wei Yang, Qin Li, Xiaoya Wang, et al. Complex Operation Path Optimization of Mother-son Shuttle Truck-type Three-dimensional Warehouse. Packaging Engineering, 40 (07): 134-141 (2019).

6. Khojasteh-Ghamari Y, Son J D, Korea J. Order Picking Problem in a Multi-Aisle Automated Warehouse Served by a Single Storage/Retrieval Machine. International Journal of Information and Management Sciences, 19(4):651-665 (2008).

7. Chaoyong Zhang, Yunqing Rao, Peigen Li, et al. An improved genetic algorithm for Job-Shop scheduling. Computer Integrated Manufacturing Systems, 10(8):966-970 (2004).

8. Luo Keen, Shougui Zhong, Changqing Wu. AVS/RS Location Optimization Based on Discrete Particle Swarm Optimization. Journal of Xiamen University (Natural Science Edition), 48 (02): 21215 (2009).

9. Mishra N, Kumar V, Kumar N, et al. Addressing Lot Sizing and Warehousing Scheduling Problem in Manufacturing Environment. Expert Systems with Applications, 38(9):11751-11762 (2011).

10. Ascheuer Norbert, Grotschel Martin, Abdel-Aziz, et al. Order Picking in an Automatic Warehouse: Solving Online Asymmetric TSPs. Mathematical Methods of Operations Research, 49(3): 501-515 (1999).

11. Wenxue Ran, Huijuan Liu. Route Optimization for Multi-objective Picking of Shuttle Trucks and Goods to People in Dynamic Three-dimensional Warehouse. Logistics Engineering and Management, 39 (06): 6973 (2017). 